\title{
A novel mobile user interface to support pedestrian navigation without restricting the user to a predefined route
}

\author{
Haosheng Huang ${ }^{\mathrm{a},}$ *, Thomas Mathis ${ }^{\mathrm{b}}$, Robert Weibel ${ }^{\mathrm{b}}$ \\ ${ }^{a}$ Department of Geography, Ghent University, Ghent, Belgium, haosheng.huang@ugent.be \\ ${ }^{b}$ Department of Geography, University of Zurich, Zurich, Switzerland, mathis.thomas@bluewin.ch, robert.weibel@geo.uzh.ch \\ * Corresponding author
}

Keywords: Location Based Services, pedestrian navigation, mobile cartography, spatial knowledge acquisition, user experience

\begin{abstract}
:
In recent years, mobile navigation systems, the most popular type of location based services, have become popular not only in outdoor environments, such as urban areas, but also in complex indoor environments, such as hospitals, university buildings, shopping malls, and airports.

State-of-the-art mobile pedestrian navigation systems often employ GPS or other positioning methods for continuous tracking of users, and thus provide them with in-situ turn-by-turn route guidance along a desired route. However, studies have shown that user experience and acquisition of spatial knowledge decrease due to the "blind" following of such turn-by-turn navigation systems. This presentation summarizes a recent study addressing these aspects. The study aimed to explore how mobile pedestrian navigation systems can be developed without restricting the user to a predefined route, and evaluates how such a system performs compared to conventional turn-by-turn systems in terms of spatial knowledge acquisition and user experience.
\end{abstract}
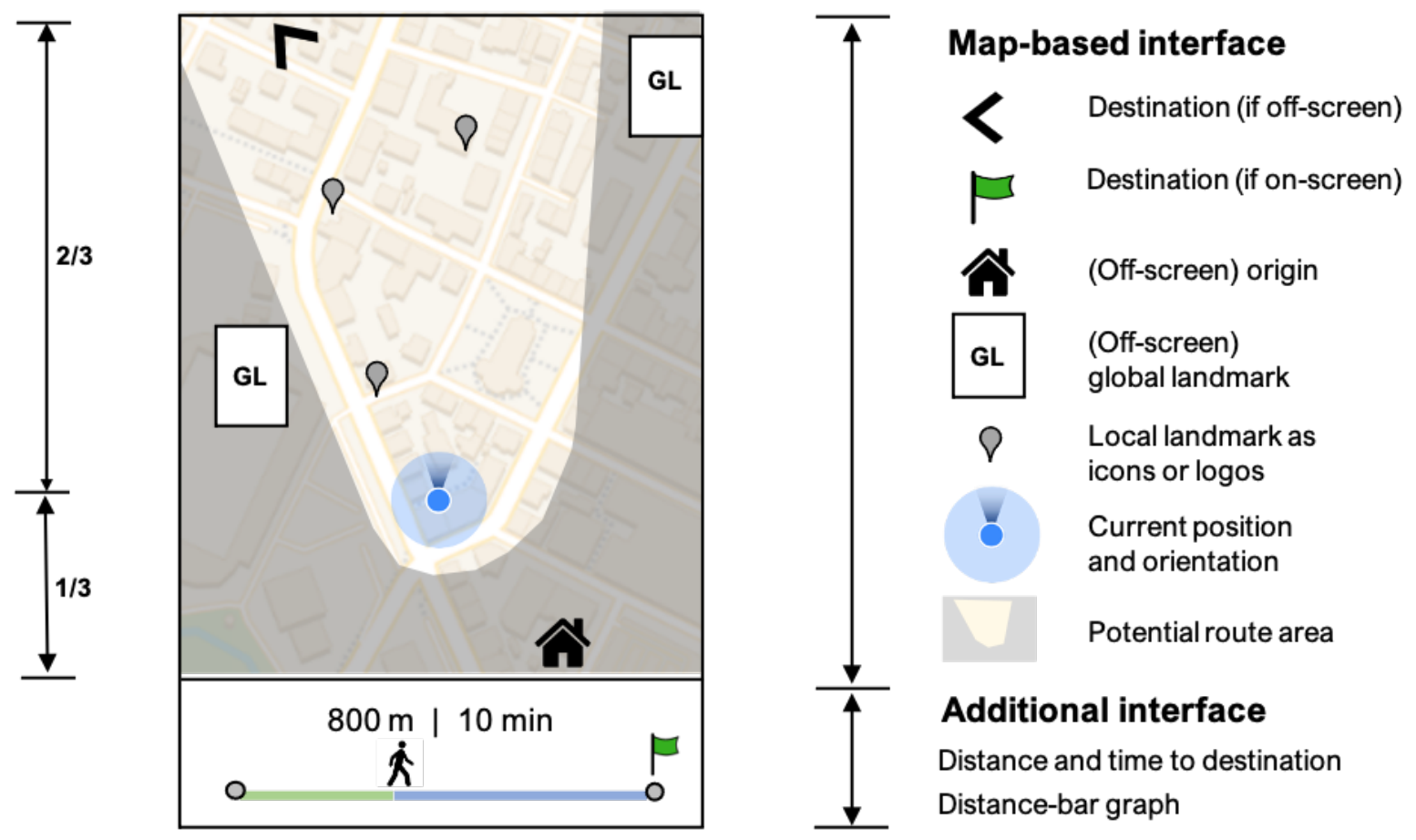

\section{Current position \\ and orientation}

Potential route area

\section{Additional interface}

Distance and time to destination

Distance-bar graph

Figure 1. Illustration of the mobile user interface, with all the interface components

A novel user interface (Figure 1) was proposed relying on the concept of Potential Route Area (PRA), which defines a dynamic area consisting of all potential routes not longer than a certain detour the user is willing to accept. Within that area, the user can freely choose his/her own route and alter it anytime, and can still arrive at the destination within the desired detour threshold. The PRA-based system was then tested against the commonly used turn-by-turn navigation system Google Maps in a real-world navigation experiment, which revealed that the acquisition of spatial knowledge and user experience were substantially improved when using the PRA-based system. 